\title{
On the fractal nature of nervous cell system
}

\author{
Gabriele Angelo Losa ${ }^{1,2 *}$, Antonio Di leva ${ }^{2,3,4,5}$, Fabio Grizzii ${ }^{2,5}$ and Gionata De Vico ${ }^{2,6}$ \\ 1 Institute of Scientific Interdisciplinary Studies, Locarno, Switzerland \\ 2 Virtual Fractal Lab (http://www.fractal-lab.org/) \\ ${ }^{3}$ Department of Systematic Anatomy, Center for Anatomy and Cell Biology, Medical University of Vienna, Vienna, Austria \\ ${ }^{4}$ Department of Neurosurgery, Medical University of Vienna, Vienna, Austria \\ 5 IRCCS Istituto Clinico Humanitas, Rozzano, Milano, Italy \\ ${ }^{6}$ Department of Biological Sciences, Faculty of MM.FF. NN. Sciences, Napoli University Federico II, Napoli, Italy \\ ${ }^{*}$ Correspondence: email: info@fractal-lab.org
}

In a detailed study entitled "Morphological development of thick - tufted layer V pyramidal cells in the rat somatosensory cortex," an international team of scientists (Romand et al., 2011) reported a series of results pertaining to an analytical investigation of the morphological development of thick-tufted layer $\mathrm{V}$ pyramidal cells (also called the principal cells) in the rat somatosensory cortex. At the end of the Introduction Section, the Authors stated "all compartments of a TTL5 cell undergo different developmental changes, supporting the notion that multiple functional compartments receive different inputs and may integrate distinct signal transduction systems." Following on a careful reading of this stimulating report a main question rose which concerned the epistemic view adopted by the Authors and in turn the analytical procedure chosen for investigating neural cells from an highly organized system, privileging in fact the recourse to "conventional" morphometry. These morphometric approaches are usually termed conventional because being based on single scale measuring which may suite well for evaluating biological objects assumed to be or arbitrary approximated to regular Euclidean structures, but inappropriate to quantitatively describe the morphology of thick-tufted layer V pyramidal cells, characterized by complex functional properties and irregular morphological features. Therefore an objective estimation could be reached only by applying the principles and rules of the Fractal geometry proposed by the mathematician Mandelbrot (1982) in the early 1980s. The Authors specified that most neural parameters, including lengths and diameters of individual segments, surface area, branch angles, and other cellular elements were "subjectively classified" and thereafter analyzed either from reconstructed figures or obtained from unrealistic representations. Another incongruous sentence was found in the Somatic Development Section: "Somata were subjectively classified into three formats according to shape: triangular, round, and oval. Although three shapes were found at all ages, somata of TTL5 neurons appeared to be mostly triangular or round at P7 and predominantly triangular thereafter." It is by far evident from Figures 1 and 3 of Romand et al. (2011) that somata, dendrites and axons are neither round or triangular bodies, nor linear segments, but appeared as irregularly shaped anatomical entities susceptible to be adequately investigated by the "non-conventional" fractal morphometry. Suffices it to mention that, during the last two decades, several studies have been performed on brain tissue and nervous system cells by adopting fractal concepts and methods, which has enabled to quantitatively elucidate most developmental, morphological, and spatial pattern avoiding arbitrary approximation or smoothing of cellular shapes and structures. (Smith and Bejar, 1994; Smith et al., 1996; Bernard et al., 2001; Grizzi and Chiriva-Internati, 2005; Milosevic and Ristanovic, 2006; Ristanovic et al., 2006; Di Ieva et al., 2007; Jelinek et al., 2008; Di Ieva, 2011). Therefore, it may not be surprising that the Authors, despite a huge investigative effort, were obliged to recognize a frank blank, honestly admitted, when they were trying to interpret the data in the light of Methodological considerations (Page 20), with the words: "Variations in results across different studies can be due to many methodological factors such as differences in the staining procedure, the section thickness, the measuring, and analyzing method, the cell selection criterion, the sample size, and the cortical area. These differences make it difficult to directly compare results between different studies." Proper considerations indeed, but not unpublished, because they evoked considerations much similar reported as far as thirty years ago by a Swiss Group (Paumgartner et al., 1981) in a pioneer study which clearly demonstrated the influence of resolution scale, i.e., objective magnification, on the estimates of geometric irregular features of liver cell membranes, or in other words the role of resolution scale at which the measurements were performed. The large observed discrepancy was consistently annulled while the variations reported by different investigators could be explained by taking into account the "resolution effect" according to the concepts of the Fractal geometry, such as the irregularity, the statistical self-similarity, the scale invariance of form, the occurrence of repetitive morphologic determinants and the fractal, i.e., non-integer dimension, rather than the trivial methodological factors called upon to explain estimate variations across different studies. Biologic structures with irregular shape and complex morphology should not be approximated to ideal geometric objects, since far from the real pictures, while a single scale of measurements should not be adopted a priori if an objective morphological description of complex objects has to be achieved (Losa and Nonnenmacher, 1996). It should be pointed out that fractal and conventional morphometric approaches, built up on distinct epistemological principles, may set the understanding of the biologic reality at different level. The former describes the morphological complexity within an experimental interval of observation scales that obviously encompasses the Euclidean dimension, while the latter proceeds at a primary level, i.e., by reducing cellular shapes and tissue structures to monotone elements which could be described by means of deterministic rules. Nevertheless, fractal and conventional morphometry may represent complementary analytical/ 
quantitative tools to elucidate the diversity of morphological patterns and functional parameters which characterize neural cells and brain structures.

\section{REFERENCES}

Bernard, F., Bossu, J. L., and Gaillard, S. (2001). Identification of living olygodendrocyte developmental stages by fractal analysis of cell morphology. J. Neurosci. Res. 65, 439-445.

Di Ieva, A. (2011). Angioarchitectural heterogeneity in human glioblastoma multiforme: a fractal- based histopathological assessment. Microvasc. Res. 81, 222-230.

Di Ieva, A., Grizzi, F., Ceva-Grimaldi, G., Russo, C., Gaetani, P., Aimar, E., Levi, D., Pisano, P., Tancioni, F., Nicola, G., Tschabitscher, M., Dioguardi, N., and Baena, R. R. (2007). Fractal Dimension as a quantitator of the microvasculature of normal and adenomatous pituitary tissue. J. Anat. 211, 673-680.

Grizzi, F., and Chiriva-Internati, M. (2005). The complexity of anatomical systems. Theor. Biol. Med. Model. 2, 26-35.
Jelinek, H. F., Milosevic, N. T., and Ristanovic, D. (2008). Fractal dimension as a tool for classification of rat retinal ganglion cells. Bioforum 101, 146-150.

Losa, G. A., and Nonnenmacher, T. F. (1996). Selfsimilarity and fractal irregularity in pathologic tissues. Mod. Pathol. 9, 174-182.

Mandelbrot, B. B. (1982). The Fractal Geometry of Nature. San Francisco: Freeman.

Milosevic, N. T., and Ristanovic, D. (2006). Fractality of dendritic arborization of spinal cord neurons. Neurosci. Lett. 396, 172-176.

Paumgartner, D., Losa, G. A., and Weibel, E. R. (1981). Resolution effect on the stereological estimation of surface and volume and its interpretation in terms of fractal dimensions. J. Microsc. 121, 51-63.

Ristanovic,D.,Stefanovic, B.D., Milosevic, N.T., Grgurevic, M., and Stankovic, J. B. (2006). Mathematical modelling and computational analysis of neuronal cell images: application to dendritic arborization of Golgiimpregnated neurons in dorsal horns of the rat spinal cord. Neurocomputing 69, 403-442.

Romand, S., Wang, Y., Toledo-Rodriguez, M., and Markram, H. (2011). Morphological development of thick-tufted layer $V$ pyramidal cells in the rat somatosensory cortex. Front. Neuroanat. 5:5. doi: 10.3389/ fnana.2011.00005

Smith, T. G. Jr., and Bejar, T. N. (1994). Comparative fractal analysis of cultured glia derived from optic nerve and brain demonstrated different rates of morphological differentiation. Brain Res. 634, 181-190.

Smith, T. G. Jr., Lange, G. D., and Marks, W. B. (1996). Fractal methods and results in cellular morphology: dimensions, lacunarity, and multifractals. J. Neurosci. Methods 69, 123-136.

Received: 14 June 2011; accepted: 08 July 2011; published online: 21 July 2011.

Citation: Losa GA, Di Ieva A, Grizzi F and De Vico G (2011) On the fractal nature of nervous cell system. Front. Neuroanat. 5:45. doi: 10.3389/fnana.2011.00045

Copyright $\odot 2011$ Losa, Di Ieva, Grizzi and De Vico. This is an open-access article subject to a non-exclusive license between the authors and Frontiers Media SA, which permits use, distribution and reproduction in other forums, provided the original authors and source are credited and other Frontiers conditions are complied with. 